\title{
Third International Symposium for Health Professionals in Rheumatology
}

\author{
Enschede 6-9 June 1990
}

At the third International Symposium for Health Professionals in Rheumatology organized by Dr. J.J. Rasker, Department of Rheumatology, Medisch Spectrum, Twente, the Netherlands, held on June 6-9 1990, the following abstracts were submitted.

\section{Plenary session I : Patient education}

\section{I.1. Old Myths and New Thinking.}

K. Lorig, Stanford University, HRP BLD6 6 Stanford, CA 94305, USA.

Traditionally, patient education is based on two myths: 1) increases in patients' knowledge lead to changes in behavior and 2) changes in behavior (exercise, pain management techniques) improve health status (pain, disability, depression).

There it little evidence in the arthritis patient education literature that changes in knowledge or behavior improve patients' health status. In fact, little association has been found between these changes and improved health status.

Explanation: The mechanism by which patient education improves arthritis may be more psychological (giving patients a sense of control) than behavioral. For example, strong associations have been shown between improved self-efficacy for controlling arthritis symptoms and health status.

Therefore, arthritis patient education programs should be designed with an emphasis on giving patients a sense of control rather than on increasing knowledge or the practice of new behaviors.

\section{I.2. Patient eduction : Selection of patient educa- tional strategies}

E. Seydel, E. Taal ${ }^{*}$, H. Rasker** . ${ }^{*}$ University of Twente; ${ }^{* *}$ Medisch Spectrum Twente, Department of Psychology, P.O. Box 217, 7500 AE Enschede, The Netherlands.
In the last few years patient education on rheumatology is a tremendous growing field of research. It is receiving more interest from behavioral scientists, physicians, nurses, policy makers, and other people who want their patients to become more informed about their conditions, to use self-management strategies, and to prevent disability. In shaping patient education health professionals are often guided by implicit criteria, vague assumptions or by trial and error. Some of them tend to equate patient education with such information dissemination techniques as teaching, and distribution of instructional pamphlets. However, instructional techniques to increase patients' knowledge is not sufficient to change behavior. There is a growing evidence, that if patient education succeeds in influencing behavior, this will not automatically result in better self-management or better health status. Self-management is defined as a process whereby a patient functions on his/her own behalf in health enhancing behavior, disease detection and treatment'. Self-efficacy seems to be an important moderator in enhancing self-management and refers to the expectation of a person that the can perform a given behavior successfully. We suggest the use of a more systematic and encompassing paradigm based on the self-efficacy concept. This concept has been successfully applied to a broad range of clinical problems, including a.o. chronic illness and health promotion. To facilitate the shaping of a patient education program based on the self-efficacy paradigm, we will present a model of patient education analysis and a set of criteria for developing and evaluating patient education programs. Some of the criteria are (1) a firm problem analysis (2) the encouragement of client responsibility, (3) full disclosure of information pertaining to the illness, (4) training of the patient in decision making skills, (5) training of the patient in generalizing skills (6) use of models or peer educators, (7) training of health professionals 
to encourage selfmanagement attitudes and behaviors of their patients.

In patient education practice, however, it is not always possible or necessary to meet all the criteria. This does not mean that a program is less valuable. The necessity to meet some of the criteria is dependent on thorough problem analysis, the objectives of the patient education and available resources (manpower, financial support). However, the model of patient education and the criteria we will present can be a useful tool in evaluating and adjusting existing programs or in developing new programs.

\section{I.3. Coping with pain- group therapy}

H.D. Basler, Institute for Medical Psychology, Bunsenstr. 3, 3550 Marburg, West Germany.

Although numerous research has been initiated by Alexander's early assumption as to the importance of psychological variables for the onset of rheumatoid arthritis, the available evidence does not support psychogenetic theories for the explanation of rheumatic diseases. On the other hand, rheumatic patients very often suffer from chronic pain and impairment and show psychological reactions as a consequence of their physical condition. Recently, the use of group therapy aimed at enhancing patients' ability to cope with disease-related stressful events has become widely accepted in behavioural medicine with a focus on pain-management procedures. Strategies for the improvement of coping with pain are based on behavioural, psychophysiological, and cognitive principles. The behavioural view conceptualizes pain as a behavioural problem with regard to facial and bodily expressions of pain, decreased physical and mental activity, and the consumption of pain medication. Operant conditioning is used to discourage pain behaviour and reinforce well-behaviour. The physiological concept stresses the vicious cycle of pain, increased muscle tension, and emotional reactions. Relaxation procedures are introduced in order to reduce excessive muscular activity in targeted muscles. The cognitive approach emphasises the effect of information-processing on pain experience. Cognitive distortions are identified, and self-control management is encouraged. Having taken all of these aspects into consideration, we developed a cognitive-behavioural treatment pro- gramme in a group setting format with components of relaxation, cognitive restructuring, and the promotion of well-being. Subjects included in the study were given diagnoses of low back pain, tension headache, rheumatoid arthritis, and ankylosing spondylitis. Treatment effects in different diagnostic groups were compared to each other, supporting the assumption that pain reduction is greatest in low back pain and least in ankylosing spondylitis. Subjects with inflammatory rheumatic diseases showed some improvement in self-reported physical complaints and in their feelings of well-being.

\section{Poster session I: Patient education}

\section{I.4. Coping and pain sensation in the evaluation of disease modifying drug treatment in rheuma-} toid arthritis.

H. Geidel, G. Leuschner, Berzirkskrankenhaus Friedrichstadt, Friedrichstraße 41, 8010 Dresden, DDR.

In a pilot study with a prospective study design we analyzed psychological reactions (using questionaire for patients) as well as aspects of pain (after pain diaries).

We examined 105 in patients with definite or classical RA at the beginning of disease modifying drug treatment and four and eight months later. The disease modifying drug treatment take place with tauredon, sulphasalazin and methotrexat (in each case 35 patients). The main questions are the interaction between disease modifying drugs and coping styles and the influence of this interaction on explorated pain reactions. The statistical analyses show interesting relations.

Finally, our results show that pain can be reduced or at least moderated and that psycho-social patient informations should be offered the necessary medical treatment of rheumatic diseases. They must bu completed with psycho-social treatments.

\section{I.5. Exercise-therapy for the cervical spine in rheumatoid arthritis.}

R.L.P. Verreusel West Mijnstr. 24H5 Sittard PO Box 5500, The Netherlands.

The aim of the physiotherapeutical approach of patients with rheumatoid arthritis (RA) is to 